\title{
RESISTANCE AT NO COST: THE TRANSMISSIBILITY AND POTENTIAL FOR DISEASE PROGRESSION OF DRUG-RESISTANT M. TUBERCULOSIS
}

Authors:

1. Mercedes C. Becerra, Sc.D., Harvard Medical School, Department of Global Health and Social Medicine, Boston, Massachusetts, United States

2. Chuan-Chin Huang, Sc.D., Brigham and Women's Hospital, Division of Global Health Equity, Department of Medicine, Boston, Massachusetts, United States

3. Leonid Lecca, M.D., Socios En Salud, Lima, Peru

4. Jaime Bayona, M.D., World Bank, Washington, District of Columbia, United States

5. Carmen Contreras, M.P.H., Socios En Salud, Lima, Peru

6. Roger Calderon, M.S., Socios En Salud, Lima, Peru

7. Rosa Yataco, B.S., Socios En Salud, Lima, Peru

8. Jerome Galea, Ph.D., Harvard Medical School, Department of Global Health and Social Medicine, Boston, Massachusetts, United States

9. Zibiao Zhang, M.S., Brigham and Women's Hospital, Division of Global Health Equity, Boston, Massachusetts, United States

10. Sidney Atwood, B.A., Brigham and Women's Hospital, Division of Global Health Equity, Boston, Massachusetts, United States

11. Ted Cohen, D.P.H., Yale School of Public Health, Epidemiology of Microbial Diseases, New Haven, Connecticut, United States

12. Carole D. Mitnick, Sc.D., Harvard Medical School, Department of Global Health and Social Medicine, Boston, Massachusetts, United States

13. Paul Farmer, M.D., Harvard Medical School, Department of Global Health and Social Medicine, Boston, Massachusetts, United States and Brigham and Women's Hospital, Division of Global Health Equity, Boston, Massachusetts, United States

14. Megan Murray, M.D., Harvard Medical School, Department of Global Health and Social Medicine, Boston, Massachusetts, United States and Brigham and Women's Hospital, Division of Global Health Equity, Boston, Massachusetts, United States

Co-corresponding authors: Megan Murray, Harvard Medical School, 641 Huntington Avenue, Boston, MA 02115 megan.murray.epi@gmail.com, 617432-2781 and Mercedes Becerra, Harvard Medical School, 641 Huntington Avenue, Boston, MA 02115 mercedes becerra@hms.harvard.edu, 617-4322540. 


\begin{abstract}
Background

The future trajectory of drug resistant tuberculosis strongly depends on the fitness costs of drug resistance mutations. Here, we measured the association of phenotypic drug resistance and the risk of TB infection and disease among household contacts (HHCs) of patients with pulmonary TB.
\end{abstract}

\title{
Methods
}

We evaluated $12767 \mathrm{HHCs}$ of patients with drug sensitive and resistant pulmonary TB at baseline, two, six, and 12 months to ascertain infection status and to determine whether they developed tuberculosis disease. We also assessed the impact of drug resistance phenotype on the likelihood that a TB strain shared a genetic fingerprint with at least one other TB patient in the cohort.

\section{Findings}

Among 3339 TB patients for whom were DST available, 1274 (38\%) had TB that was resistant to at least one drug and $478(14.3 \%)$ had multi-drug resistant (MDR) TB, i.e. TB resistant to both INH and rifampicin. Compared to HHCs of drug sensitive TB patients, those exposed to a patient with MDR-TB had an $8 \%$ $(95 \% \mathrm{Cl}: 4-13 \%)$ higher risk of infection by the end of follow up. We found no statistically significant difference in the relative hazard of incident TB disease among HHCs exposed to MDR-TB compared to DS-TB (Adjusted HR $1 \cdot 28$ [(95\% Cl: $\cdot 9-1 \cdot 83])$. Patients with MDR-TB were more likely to be part of a genetic cluster than were DS-TB patients.

\section{Interpretation}

Clinical strains of MDR M. tuberculosis are neither less transmissible than drug sensitive strains nor less likely to cause disease. (ClinicalTrials.gov number, NCT00676754)

\section{Funding}

National Institutes of Health: NIH/NIAID CETR U19AI109755

\section{Statement}

All authors have seen the manuscript and approved the manuscript. 
Resistance to tuberculosis (TB) drugs threatens to slow gains against the global TB pandemic. In 2015, of $10 \cdot 4$ million new TB cases, 500,000 were caused by MDR-TB, i.e., TB resistant to both isoniazid and rifampicin. Access to effective treatment for MDR-TB remains limited, and cure is achieved in only $50 \% .^{1}$

The primary strategy deployed against MDR-TB was designed to reduce the emergence of new resistance but did not address existing drug resistant strains. This policy focused on the empirical treatment of presumed drugsensitive TB (DS-TB) to reduce the acquisition of drug-resistance in individual patients during suboptimal therapy. ${ }^{2,3}$ While this approach likely prevents patients from acquiring new drug resistance, it does nothing to interrupt the transmission of existing DR-TB strains. ${ }^{4}$ With MDR-TB prevalence in some regions as high as $32 \%,{ }^{1}$ the course of the epidemic will depend on how quickly DR-TB patients are detected and rendered non-infectious as well as on the relative transmissibility of DR-TB strains. ${ }^{5-7}$ If the acquisition of drug-resistance mutations by Mycobacterium tuberculosis (Mtb) incurs a "fitness cost" that reduces its ability to spread, the incidence of DR-TB would be expected to decline more slowly than if resistance were cost-free..$^{7-9}$ The projections of mathematical models forecasting TB incidence strongly depend on this putative cost. $^{7}$

We undertook to estimate the fitness cost of drug resistance by comparing the rates of TB infection and disease among individuals exposed to DR-TB and DS-TB. We further measured the association between drug resistance profile (DRP) and whether strains were members of genetic clusters.

\section{METHODS}

\section{Study population and setting}

This study was conducted in a catchment area of Lima, Peru that included approximately 33 million residents. Detailed descriptions of recruitment and enrollment procedures are provided in the Supplement. The study protocol was approved by the Harvard University Institutional Review Board and by the Research Ethics Committee of the National Institute of Health of Peru.

\section{Index patient assessment}

Briefly, we identified adults $>=15$ years who presented with clinically suspected pulmonary TB at 106 participating health centers from September 2009 through August 2012. Two sputum samples were evaluated by Ziehl-Neelsen (AFB) and mycobacterial culture.

At enrollment, we collected information on age, gender, socio-demographics, BCG vaccination, alcohol and tobacco use, comorbid disease, previous TB 
disease, duration of symptoms, presence of cavitary disease on chest radiograph, sputum smear status, culture results, HIV status, and CD4 count. For those with positive TB cultures, isolates underwent drug-susceptibility testing (DST) and MIRU-based genotyping.

Because DST results are not routinely available until two or more months after patients start therapy, those with unsuspected DR-TB received ineffective regimens prior to the receipt of DST results and would thus be expected to remain infectious during that period. We therefore also recorded the time from enrollment until the initiation of effective therapy (See Supplement).

\section{Enrollment of household contacts}

Within two weeks of enrolling an index patient, we invited his or her household contacts (HHCs) to participate in this longitudinal study. We screened consenting HHCs for TB signs and symptoms; those who screened positive were referred to a health center for routine evaluation and treatment.

\section{Assessment of household contacts}

At enrollment, we obtained the following information: age, gender, sociodemographics, BCG vaccination, alcohol and tobacco use, comorbid disease, concurrent medication use, TB disease history, use of isoniazid preventive therapy (IPT), height, weight, HIV status, and CD4 count. In HHCs with no history of TB, we obtained a tuberculin skin test (TST). We considered HHCs to be TB infected if they had a previous history of TB or a TST induration size $>10$ $\mathrm{mm}$ in non-HIV infected contacts and $>5 \mathrm{~mm}$ in HIV-infected contacts.

We evaluated all HHCs for pulmonary and extra-pulmonary TB disease at two, six, and 12 months after enrollment. HHCs diagnosed with TB disease were classified as having either co-prevalent TB (disease diagnosed within 14 days of the index patient) or secondary TB (disease diagnosed after 14 days). We defined TB disease in $\mathrm{HHCs}<18$ years according to consensus guidelines for diagnosis of TB in children. ${ }^{10} \mathrm{HHCs}$ who were previously TST negative were retested at six and 12 months. We considered HHCs to have become infected during follow-up if they had a negative TST at any point in the study that was followed by an increase in TST induration size of at least $6 \mathrm{~mm}$.

\section{Statistical Analysis}

\section{TB transmission}

To determine whether DRP of the index patient was an independent risk factor for TB infection among HHCs, we used modified Poisson regressions to assess the prevalence odds of infection by the end of follow-up, thereby capturing infection events that occurred both prior to and after the diagnosis of the index 
patient. In sensitivity analyses, we assessed the association between DRP and TST positivity at enrollment, and used Cox frailty proportional hazards models to assess the association between DRP and the risk of becoming infected during follow up. We estimated the time of infection as the mid-point between the last negative TST and either the first positive TST or a diagnosis of TB disease. We considered the possibility that the impact of DRP was mediated through its effect on either the clinical presentation of TB in the index patient (sputum smear positivity or cavitary lesions) or through prolonged infectiousness due to a delay in receipt of effective treatment. To assess the direct effect of DRP on infection, i.e. the effect that is not mediated through these intermediates, we compared models with and without adjustment for these factors. Because we considered that children who were infected at baseline were more likely to have been infected by the corresponding index patient rather than during a prior exposure, we also conducted sensitivity analyses that restricted the population of HHCs to children $<15$.

\section{TB disease incidence}

To assess the impact of DRP on the risk of secondary TB disease in HHCs, we used a Cox frailty proportional hazards model, adjusting for factors known or hypothesized to affect TB disease risk. We also estimated this effect with and without adjustment of the possible mediators cited above. We conducted sensitivity analyses that included only secondary cases who shared an identical or similar MIRU pattern with the index patient.

\section{TB clustering}

We assessed the impact of DRP on the genetic clustering of TB cases, under the assumption that clustering is a proxy for transmission and disease progression. We classified Mtb isolates as clustered if they shared a 24-locus MIRU pattern with an isolate from at least one other case in the study. We then used logistic regression to estimate the impact of DRP on propensity to cluster.

\section{Role of the funding source}

The funder of the study had no role in study design, data collection, data analysis, data interpretation, or writing of the report. The corresponding author had full access to all study data and had final responsibility for the decision to submit for publication.

\section{RESULTS}

Between September 2009 and September 2012, we enrolled 4,500 index patients, of whom 4,044 had microbiologically confirmed TB disease (Table S1). Of the 3,339 for whom DSTs were available, $1274(38 \%)$ had isolates resistant to at least one drug: $538(16 \cdot 4 \%)$ to only one drug and $478(14 \cdot 3 \%)$ to 
both isoniazid and rifampicin (MDR-TB). The proportion of patients who had MDR-TB decreased with age, ranging from $34 \cdot 1 \%$ among those $0-15$ years of age to $6 \cdot 2 \%$ in those over 60 . We enrolled 10,160 household contacts of the 2,563 microbiologically confirmed index patients who had a DTS available and at least one $\mathrm{HHC}$ (Table 1).

\section{TB infection}

At enrollment, 4,488 (44.2\%) HHCs were TB-infected. Compared to HHCs of DS-TB index patients, those exposed to INH mono-resistant TB had a $15 \%$ (95\% Cl: $5 \%-16 \%$ ) higher risk of infection by 12 months, while those exposed to MDR-TB had an $8 \%(95 \% \mathrm{Cl}: 4-13 \%)$ higher risk (Table 2$)$. In sensitivity analyses, the positive association between exposure to both INH monoresistant TB and MDR-TB and the risk of infection remained similar when we assessed the prevalence ratio of infection at baseline (Table S2), the hazard of TST conversion during follow up (Table S3), when we restricted all of the above analyses to children, and when we estimated the direct effect of DRP on infection by controlling for smear status, cavitary disease, treatment delay, and time to effective treatment (Tables S4-S6).

\section{TB disease}

There was no statistically significant difference in the relative hazard of incident TB disease among HHCs exposed to DR-TB compared to DS-TB (Adjusted HR for INH mono-resistant $\cdot 17$ [95\% Cl: $\cdot 02-1 \cdot 26])$ and for MDR-TB 1·28,[(·9-1·83] (Table 3 and Figure S1). For MDR-TB, this result persisted when we only considered secondary cases if the molecular fingerprint matched that of the corresponding index patient and when matches were based on either more or less stringent criteria (Table 4).

\section{TB clustering}

Among 3,608 Mtb isolates from unique individuals, $74 \%$ were clustered by MIRU genotyping and $26 \%$ did not match any other genotype in the dataset (Figure S2). Table 5 shows the relative risk of clustering by potential risk factors for transmission; in both univariate and adjusted analyses, patients with either INH mono-resistant TB or MDR-TB are more likely to be part of a cluster than are DS-TB patients. 


\section{DISCUSSION}

In this large prospective cohort study of TB patient households, we observed that individuals exposed to MDR-TB patients are at higher risk of becoming infected with TB when compared to those exposed to DS-TB and at similar risk of developing TB disease during the 12 months following the diagnosis of the index patient. These data suggest that, compared to DS-TB, MDR-TB is no less transmissible and no less likely to lead to disease. We also found that DR-TB patients are more likely than those with DS-TB to be part of a cluster as defined by a matching MIRU-based genotype. Because clustered patients are presumed to be linked through recent transmission followed by disease progression, ${ }^{11}$ these results support the conclusion that drug resistance does not incur fitness costs in the DR-TB strains circulating in this setting. This hypothesis is also consistent with our observation that the proportion of TB patients who were MDR was highest among the youngest groups, i.e., those most likely to be recently infected.

As the largest study to date of the relative transmissibility and risk of disease progression of DR-TB, our study adds to a diverse body of work on the fitness cost of Mtb drug resistance. Laboratory studies that have assessed the fitness cost of drug resistance by comparing bacterial growth rates in media and/or bacillary loads and survival in infected animal models indicate that, ${ }^{12-14}$ while some resistance-causing mutations reduce growth rates or virulence, ${ }^{15-17}$ others have little or variable impact. ${ }^{18-20}$ Even when mutations do confer fitness costs, subsequent "compensatory" mutations can reverse these growth defects while preserving the resistance phenotypes. ${ }^{21,22}$ As would be expected, such low-cost or compensatory mutations are observed at higher frequency than other resistance mutations. ${ }^{23,24}$

Studies of the relative fitness of DR-TB in human populations have approached the question in two ways: by either assessing the effect of DRP on the relative frequency of clustered compared to unclustered cases or by directly measuring the risk of infection and disease among contacts of DS-TB and DR-TB patients. ${ }^{5}$ Multiple molecular epidemiologic studies show that DR-TB strains can be transmitted and that clusters of resistant strains can persist over long periods. ${ }^{25-28}$ However, previous molecular epidemiologic studies have reached different conclusions; some have found that DR strains are more likely to be clustered than DS strains while others have shown the reverse. ${ }^{29-31}$ Some studies have suggested that the association with clustering depends on the specific drug resistance phenotype and/or mutation. ${ }^{32,33}$ Many of these previous studies have been small and subject to biases due to convenience sampling of isolates. ${ }^{34,35}$ Our study, which aimed to prospectively capture all notified cases in a geographically contiguous area, followed by close follow up of household contacts for infection and incidence TB disease, is, to our knowledge, the largest study to systematically examine drug resistance as a risk factor for transmission and disease. 
The few studies to date that have directly measured the capacity of DR and DS $M t b$ strains to cause infection or disease have also yielded conflicting results. In 1984, Snider et al. reported no difference in the risk of TB infection in child household contacts exposed to either INH- or streptomycin-resistant Mtb compared to DS Mtb. ${ }^{36}$ Although the authors also reported no differences in disease incidence in these groups, the study was not powered to detect this outcome and confidence intervals were wide. Similarly, in a small 2001 study conducted in Brazil, Teixeira et al. found that household contacts of MDR-TB patients were slightly more likely to be infected at baseline than contacts of DSTB patients and equally likely to develop disease, although neither result was statistically significant. ${ }^{37}$ In 2011 , the TB Research Centre in India reported on 5,562 household contacts exposed to DS-TB and 779 exposed to INH-resistant TB. This cohort was followed for up to 15 years; the prevalence of TB infection was higher in the contacts of INH-resistant patients, while the hazard of disease was similar in the two groups. ${ }^{38}$ In contrast to these results, in a study also conducted in Peru, Grandjean et al. found that MDR-TB household contacts were half as likely to develop TB disease compared to DS-TB contacts. ${ }^{39}$ Finally, in a small study from Vietnam, Fox et al. found greater TB infection and disease among contacts of patients with known MDR-TB compared to contacts of patients with recently diagnosed TB presumed to be DS-TB (DST was not available for the second group). ${ }^{40}$ Thus, our results are consistent with the majority of previous studies on risk among household contacts, as well as with our own cluster analyses.

We note several important limitations to this study. First, because TSTs only measure previous infection but not the time of its occurrence, there is no ideal way to measure the incidence of TB infection due to a specific, time-dependent exposure. Baseline TST positivity may reflect a remote infection that occurred prior to a person's exposure to the index patient, while TST conversion among people who tested negative at baseline is subject to survival bias (see Supplement). We examined these issues by conducting multiple sensitivity analyses; all were consistent with our main results.

Secondly, although the study was designed to use molecular fingerprinting to ascertain whether transmission had taken place between an index patient and secondary case, most of the secondary cases in our cohort occurred among child contacts, most of whom did not have microbiological confirmation of TB disease. Although it is expected that a child sick with TB was most likely infected by someone in the home, it is possible that the infection resulted from community-based transmission. Within the subset of 133 secondary cases for whom a genotype was available, only $56(43.6 \%)$ matched the genotype of the index patient. Our finding of no significant difference in the hazard of disease after exposure to a DR or DS patient in this subset may reflect the small numbers rather than the absence of an effect. Interestingly, our finding that fewer than half of the genotyped household pairs shared a genotype is 
consistent with what has been reported from household contact studies in other high burden settings, where this fraction ranged from 25 to $50 \% .{ }^{38,40}$ It is unclear whether the incidence of unmatched secondary cases represents background rates of community transmission or signals particularly high levels of vulnerability to TB as a result of shared genetic or environmental risk factors.

These results have major implications for public health policy and for projecting the burden of DR-TB. Mathematical models suggest that the expected trajectories of DS-TB and DR-TB strongly depend on the fitness cost of clinically relevant resistance mutations. If $M t b$ drug resistance exacts no fitness cost, the incidence of DR and MDR TB will be expected to fall more slowly than would be expected even in populations where the acquisition of new drug resistance is minimized through measures such as supervised therapy to ensure adherence to standardized empirical regimens. ${ }^{41}$ Our findings provide strong evidence that TB programs must immediately deploy strategies that directly target DR-TB and MDR-TB, such as the early detection and effective treatment of both infection and disease. These should include both the wider deployment of existing tools and the further development of diagnostic and therapeutic strategies designed specifically for persons already infected with DR-TB.

\section{AUTHOR CONTRIBUTIONS}

Lima Cohort Study: MBM, MCB, TC, CM and PEF conceived of the study and $M B M$ and MCB led the study design. LL oversaw data collection and management with JB, JTG, RC, ZZ, CC, SA and RY. RC managed laboratory efforts. MBM supervised data analysis and interpretation in conjunction with $\mathrm{MCB}$ and $\mathrm{CCH}$.

MCB and MBM wrote the first draft of the manuscript, and all authors contributed to manuscript revision.

\section{DECLARATION OF INTERESTS}

We declare no competing interests.

\section{ACKNOWLEDGEMENTS}

This study was funded by National Institutes of Health (NIH/NIAID CETR U19AI109755). 


\section{References}

1. WHO Tuberculosis Report 2016

(http://ps.who.int/iris/bitstream/10665/250441/1/9789241565394-

eng.pdf?ua=1) Accessed 24 March 2018.

2. Weis SE, Slocum PC, Blais FX, et al. The effect of directly observed therapy on the rates of drug resistance and relapse in tuberculosis. $N$ Engl $J$ Med 1994;330:1179-1184.

3. Loddenkemper R, Sagebiel D, Brendel A. Strategies against multidrugresistant tuberculosis. Eur Respir J Supp/ 2002;36:66s-77s.

4. Horsburgh CR Jr. The global problem of multidrug-resistant tuberculosis: the genie is out of the bottle. JAMA 2000;283:2575-6.

5. Cohen T, Dye C, Colijn C, Williams B, Murray M. Mathematical models of the epidemiology and control of drug-resistant TB. Expert Rev Respir Med 2009;3:67-79.

6. Blower SM, Gerberding JL. Understanding, predicting and controlling the emergence of drug-resistant tuberculosis: a theoretical framework. $J \mathrm{Mol}$ Med (Berl) 1998;76:624-36. Review.

7. Blower SM, Chou T. Modeling the emergence of the 'hot zones': tuberculosis and the amplification dynamics of drug resistance. Nat Med 2004;10:1111-6.

8. Cohen T, Murray M. Modeling epidemics of multidrug-resistant M. tuberculosis of heterogeneous fitness. Nat Med 2004;10:1117-21. 
9. Knight GM, Colijn C, Shrestha S, et al. The Distribution of Fitness Costs of Resistance-Conferring Mutations Is a Key Determinant for the Future Burden of Drug-Resistant Tuberculosis: A Model-Based Analysis. Clin Infect Dis 2015;61:S147-54.

10. Graham SM, Ahmed T, Amanullah F, et al. Evaluation of tuberculosis diagnostics in children: 1. Proposed clinical case definitions for classification of intrathoracic tuberculosis disease. Consensus from an expert panel. $J$ Infect Dis 2012;205:S199-208.

11. Mathema B, Kurepina NE, Bifani PJ, Kreiswirth BN. Molecular epidemiology of tuberculosis: current insights. Clin Microbiol Rev 2006;19:658-85. Review.

12. Cohen T, Sommers B, Murray M. The effect of drug resistance on the fitness of Mycobacterium tuberculosis. Lancet Infect Dis 2003;3:13-21. Review.

13. Andersson DI, Levin BR. The biological cost of antibiotic resistance. Curr Opin Microbiol 1999;2:489-93. Review.

14.Pope CF, McHugh TD, Gillespie SH. Methods to determine fitness in bacteria. In: Gillespie SH, McHugh TD, eds. Antibiotic Resistance Protocols. 2nd ed. Totowa, NJ: Springer, 2010:113-121.

15. Cohn ML, Kovitz C, Oda U, Middlebrook G. Studies on isoniazid and tubercle bacilli. II. The growth requirements, catalase activities, and pathogenic properties of isoniazid-resistant mutants. Am Rev Tuberc 1954;70:852-72. 
16. Mariam DH, Mengistu Y, Hoffner SE, Andersson DI. Effect of rpoB mutations conferring rifampin resistance on fitness of Mycobacterium tuberculosis. Antimicrob Agents Chemother 2004;48:1289-1294.

17.Davies AP, Billington OJ, Bannister BA, Weir WR, McHugh TD, Gillespie $\mathrm{SH}$. Comparison of fitness of two isolates of Mycobacterium tuberculosis, one of which had developed multi-drug resistance during the course of treatment. J Infect 2000;41:184-187.

18. Pym AS, Saint-Joanis B, Cole ST. Effect of katG mutations on the virulence of Mycobacterium tuberculosis and the implication for transmission in humans. Infect Immun 2002;70:4955-4960.

19. Billington OJ, McHugh TD, Gillespie SH. Physiological cost of rifampin resistance induced in vitro in Mycobacterium tuberculosis. Antimicrob Agents Chemother 1999;43: 1866-1869.

20. Ordway DJ, Sonnenberg MG, Donahue SA, Belisle JT, Orme IM. Drugresistant strains of Mycobacterium tuberculosis exhibit a range of virulence for mice. Infect Immun 1995;63:741-3.

21. Brandis G, Pietsch F, Alemayehu R, Hughes D. Comprehensive phenotypic characterization of rifampicin resistance mutations in Salmonella provides insight into the evolution of resistance in Mycobacterium tuberculosis. $J$ Antimicrob Chemother 2015;70:680-5.

22. Comas I, Borrell S, Roetzer A, et al. Whole-genome sequencing of rifampicin-resistant Mycobacterium tuberculosis strains identifies 
compensatory mutations in RNA polymerase genes. Nat Genet 2011;44:106-10.

23. Gagneux S, Long CD, Small PM, Van T, Schoolnik GK, Bohannan BJ. The competitive cost of antibiotic resistance in Mycobacterium tuberculosis. Science 2006;312:1944-6.

24. Cohen T, Becerra MC, Murray MB. Isoniazid resistance and the future of drug-resistant tuberculosis. Microb Drug Resist 2004;10:280-5.

25. Shah NS, Auld SC, Brust JC, et al. Transmission of Extensively DrugResistant Tuberculosis in South Africa. N Engl J Med 2017;376(3):243-253.

26. Yang C, Luo T, Shen X, et al. Transmission of multidrug-resistant Mycobacterium tuberculosis in Shanghai, China: a retrospective observational study using whole-genome sequencing and epidemiological investigation. Lancet Infect Dis 2017;17:275-284.

27. Smith CM, Trienekens SC, Anderson C, et al. Twenty years and counting: epidemiology of an outbreak of isoniazid-resistant tuberculosis in England and Wales, 1995 to 2014. Euro Surveill 2017;22:30467.

28. Marais BJ, Mlambo CK, Rastogi N, et al. Epidemic spread of multidrugresistant tuberculosis in Johannesburg, South Africa. J Clin Microbiol 2013;51:1818-25.

29. García-García ML, Jiménez-Corona ME, Ponce-de-León A, et al. Mycobacterium tuberculosis drug resistance in a suburban community in southern Mexico. Int J Tuberc Lung Dis 2000;4:S168-70. 
30. van Soolingen D, Borgdorff MW, de Haas PE, et al. Molecular epidemiology of tuberculosis in the Netherlands: a nationwide study from 1993 through 1997. J Infect Dis 1999;180:726-36.

31. Toungoussova OS, Sandven P, Mariandyshev AO, Nizovtseva NI, Bjune G, Caugant DA. Spread of drug-resistant Mycobacterium tuberculosis strains of the Beijing genotype in the Archangel Oblast, Russia. J Clin Microbiol 2002;40:1930-7.

32. Gagneux S, Burgos MV, DeRiemer K, et al. Impact of bacterial genetics on the transmission of isoniazid-resistant Mycobacterium tuberculosis. PLoS Pathog 2006;2:e61.

33. Burgos M, DeRiemer K, Small PM, Hopewell PC, Daley CL. Effect of drug resistance on the generation of secondary cases of tuberculosis. J Infect Dis 2003;188:1878-84.

34. Murray M. Sampling bias in the molecular epidemiology of tuberculosis. Emerg Infect Dis 2002;8:363-9.

35. Glynn JR, Bauer J, de Boer AS, et al. Interpreting DNA fingerprint clusters of Mycobacterium tuberculosis. European Concerted Action on Molecular Epidemiology and Control of Tuberculosis. Int J Tuberc Lung Dis 1999;3:1055-60.

36. Snider DE Jr, Kelly GD, Cauthen GM, Thompson NJ, Kilburn JO. Infection and disease among contacts of tuberculosis cases with drug-resistant and drug-susceptible bacilli. Am Rev Respir Dis 1985;132:125-32. 
37. Teixeira L, Perkins MD, Johnson JL, et al. Infection and disease among household contacts of patients with multidrug-resistant tuberculosis. Int $J$ Tuberc Lung Dis 2001;5(4):321-8.

38. Tuberculosis Research Centre, Indian Council of Medical Research (ICMR), Chennai, India. Risk of tuberculosis among contacts of isoniazid-resistant and isoniazid-susceptible cases. Int J Tuberc Lung Dis 2011;15:782-8.

39. Grandjean L, Gilman RH, Martin L, et al. Transmission of MultidrugResistant and Drug-Susceptible Tuberculosis within Households: A Prospective Cohort Study. PLoS Med 2015;12(6):e1001843.

40. Fox GJ, Anh NT, Nhung NV, et al. Latent tuberculous infection in household contacts of multidrug-resistant and newly diagnosed tuberculosis. Int $J$ Tuberc Lung Dis 2017;21(3):297-02.

41. Sharma A, Hill A, Kurbatova E, et al. Estimating the future burden of multidrug-resistant and extensively drug-resistant tuberculosis in India, the Philippines, Russia, and South Africa: a mathematical modelling study. Lancet Infect Dis 2017;17:707-715. 


\section{TABLES}

Table 1

Characteristics of household contacts of patients with pulmonary TB in Lima, Peru.

\begin{tabular}{|c|c|c|c|c|c|c|c|c|}
\hline \multirow{2}{*}{$\begin{array}{l}\text { Variable } \\
\text { Age }\end{array}$} & \multicolumn{8}{|c|}{ Drug resistance profile of index patient } \\
\hline & \multicolumn{2}{|c|}{ Pan-susceptible } & \multicolumn{2}{|c|}{ Mono-resistant } & \multicolumn{2}{|c|}{ Poly-resistant } & \multicolumn{2}{|c|}{$\begin{array}{c}\text { Multidrug- } \\
\text { resistant }\end{array}$} \\
\hline 16 to 30 & 1,667 & $26 \cdot 9 \%$ & 425 & $25 \cdot 6 \%$ & 225 & $29 \cdot 2 \%$ & 443 & $28 \cdot 7 \%$ \\
\hline 31 to 45 & 1,063 & $17 \cdot 2 \%$ & 316 & $19 \cdot 0 \%$ & 130 & $16 \cdot 9 \%$ & 302 & $19 \cdot 6 \%$ \\
\hline 46 to 60 & 807 & $13 \cdot 0 \%$ & 244 & $14 \cdot 7 \%$ & 114 & $14 \cdot 8 \%$ & 174 & $11 \cdot 3 \%$ \\
\hline 61 and older & 459 & $7 \cdot 4 \%$ & 82 & $4 \cdot 9 \%$ & 60 & $7 \cdot 8 \%$ & 96 & $6 \cdot 2 \%$ \\
\hline Male & 2,814 & $45 \cdot 5 \%$ & 711 & $42 \cdot 9 \%$ & 344 & $44 \cdot 6 \%$ & 718 & $46 \cdot 6 \%$ \\
\hline HIV seropositive & 21 & $0 \cdot 3 \%$ & 4 & $0 \cdot 2 \%$ & 5 & $0 \cdot 7 \%$ & 5 & $0 \cdot 3 \%$ \\
\hline Diabetes & 110 & $1 \cdot 8 \%$ & 30 & $1 \cdot 8 \%$ & 16 & $2 \cdot 1 \%$ & 27 & $1 \cdot 8 \%$ \\
\hline \multicolumn{9}{|l|}{ BCG scars } \\
\hline 0 & 866 & $14 \cdot 0 \%$ & 211 & $12 \cdot 7 \%$ & 121 & $15 \cdot 7 \%$ & 217 & $14 \cdot 1 \%$ \\
\hline 1 & 3,994 & $64 \cdot 5 \%$ & 1078 & $65 \cdot 0 \%$ & 455 & $59 \cdot 0 \%$ & 974 & $63 \cdot 2 \%$ \\
\hline 2 & 1,053 & $17 \cdot 0 \%$ & 297 & $17 \cdot 9 \%$ & 157 & $20 \cdot 4 \%$ & 283 & $18 \cdot 4 \%$ \\
\hline$\geq 3$ & 276 & $4 \cdot 5 \%$ & 72 & $4 \cdot 3 \%$ & 38 & $4 \cdot 9 \%$ & 67 & $4 \cdot 3 \%$ \\
\hline \multicolumn{9}{|l|}{ Smoking status } \\
\hline Non-smoker & 5,745 & $93 \cdot 8 \%$ & 1528 & $93 \cdot 1 \%$ & 724 & $95 \cdot 0 \%$ & 1,443 & $94 \cdot 3 \%$ \\
\hline 1 cigarette per day & 196 & $3 \cdot 2 \%$ & 61 & $3 \cdot 7 \%$ & 22 & $2 \cdot 9 \%$ & 46 & $3 \cdot 0 \%$ \\
\hline$>1$ cigarette per day & 183 & $3 \cdot 0 \%$ & 52 & $3 \cdot 2 \%$ & 16 & $2 \cdot 1 \%$ & 41 & $2 \cdot 7 \%$ \\
\hline \multicolumn{9}{|l|}{ Alcohol use } \\
\hline Non-drinker & 4,479 & $73 \cdot 8 \%$ & 1195 & $73 \cdot 0 \%$ & 562 & $74 \cdot 5 \%$ & 1139 & $75 \cdot 8 \%$ \\
\hline 0 to $<3$ drinks per day & 1,270 & $20 \cdot 9 \%$ & 353 & $21 \cdot 6 \%$ & 145 & $19 \cdot 2 \%$ & 283 & $18 \cdot 8 \%$ \\
\hline$\geq 3$ drinks per day & 321 & $5 \cdot 3 \%$ & 89 & $5 \cdot 4 \%$ & 47 & $6 \cdot 2 \%$ & 81 & $5 \cdot 4 \%$ \\
\hline \multicolumn{9}{|l|}{ Nutritional status } \\
\hline Normal weight & 3,561 & $58 \cdot 1 \%$ & 935 & $57 \cdot 0 \%$ & 422 & $55 \cdot 2 \%$ & 881 & $57 \cdot 7 \%$ \\
\hline Underweight & 103 & $1 \cdot 7 \%$ & 22 & $1 \cdot 3 \%$ & 16 & $2 \cdot 1 \%$ & 27 & $1 \cdot 8 \%$ \\
\hline Overweight & 2,470 & $40 \cdot 3 \%$ & 683 & $41 \cdot 6 \%$ & 327 & $42 \cdot 7 \%$ & 620 & $40 \cdot 6 \%$ \\
\hline Employed & 3,931 & $63 \cdot 5 \%$ & 1019 & $61 \cdot 5 \%$ & 460 & $59 \cdot 7 \%$ & 973 & $63 \cdot 1 \%$ \\
\hline Public transportation & 2,255 & $36 \cdot 5 \%$ & 638 & $38 \cdot 5 \%$ & 311 & $40 \cdot 3 \%$ & 568 & $36 \cdot 9 \%$ \\
\hline Non-user & 1,636 & $26 \cdot 9 \%$ & 469 & $28 \cdot 8 \%$ & 181 & $24 \cdot 0 \%$ & 443 & $29 \cdot 4 \%$ \\
\hline 1 to 3 days per week & 1,969 & $32 \cdot 4 \%$ & 517 & $31 \cdot 8 \%$ & 246 & $32 \cdot 6 \%$ & 495 & $32 \cdot 9 \%$ \\
\hline 4 to 7 days per week & 2,466 & $40 \cdot 6 \%$ & 640 & $39 \cdot 4 \%$ & 328 & $43 \cdot 4 \%$ & 568 & $37 \cdot 7 \%$ \\
\hline Completed high school & 1,143 & $20 \cdot 7 \%$ & 315 & $21 \cdot 2 \%$ & 175 & $25 \cdot 0 \%$ & 247 & $17 \cdot 8 \%$ \\
\hline Socioeconomic status & & & & & & & & \\
\hline
\end{tabular}


bioRxiv preprint doi: https://doi.org/10.1101/475764; this version posted November 23, 2018. The copyright holder for this preprint (which was not certified by peer review) is the author/funder. All rights reserved. No reuse allowed without permission.

\begin{tabular}{|l|l|l|l|l|l|l|l|l|}
\hline Low & 2,125 & $35 \cdot 1 \%$ & 602 & $36 \cdot 9 \%$ & 261 & $36 \cdot 0 \%$ & 528 & $34 \cdot 5 \%$ \\
\hline Middle & 2,670 & $44 \cdot 1 \%$ & 682 & $41 \cdot 8 \%$ & 325 & $44 \cdot 9 \%$ & 728 & $47 \cdot 5 \%$ \\
\hline High & 1,261 & $20 \cdot 8 \%$ & 347 & $21 \cdot 3 \%$ & 138 & $19 \cdot 1 \%$ & 276 & $18 \cdot 0 \%$ \\
\hline
\end{tabular}




\begin{tabular}{|c|c|c|c|c|c|}
\hline Resistant to & $\begin{array}{l}\text { Prevalence of } \\
\text { infection }\end{array}$ & Univariate analysis & $\begin{array}{c}\text { Multivariate model } \\
1 *\end{array}$ & $\begin{array}{l}\text { Multivariate model } \\
2 * *\end{array}$ & $\begin{array}{c}\text { Multivariate model } \\
3^{* * *}\end{array}$ \\
\hline & Number (percent) & PR $(95 \% \mathrm{CI})$ & PR $(95 \% \mathrm{CI})$ & $\mathrm{PR}(95 \% \mathrm{CI})$ & $\mathrm{PR}(95 \% \mathrm{CI})$ \\
\hline Susceptible & $3597(58 \cdot 5)$ & Ref & Ref & Ref & Ref \\
\hline Mono-Inh & $185(3)$ & $1 \cdot 17(1 \cdot 09-1 \cdot 25)$ & $1 \cdot 16(1 \cdot 08-1 \cdot 24)$ & $1 \cdot 14(1 \cdot 07-1 \cdot 23)$ & $1 \cdot 15(1 \cdot 06-1 \cdot 24)$ \\
\hline Mono-Strep & $716(11 \cdot 6)$ & $1 \cdot 04(0 \cdot 99-1 \cdot 09)$ & $1 \cdot 03(0 \cdot 98-1 \cdot 08)$ & $1 \cdot 03(0 \cdot 98-1 \cdot 08)$ & $1 \cdot 02(0 \cdot 98-1 \cdot 08)$ \\
\hline Inh+Strep & $256(4 \cdot 2)$ & $1 \cdot 08(1 \cdot 01-1 \cdot 16)$ & $1 \cdot 06(0 \cdot 99-1 \cdot 14)$ & $1 \cdot 06(0 \cdot 99-1 \cdot 14)$ & $1 \cdot 04(0 \cdot 95-1 \cdot 12)$ \\
\hline MDR & $1041(16 \cdot 9)$ & $1 \cdot 08(1 \cdot 04-1 \cdot 13)$ & $1 \cdot 08(1 \cdot 04-1 \cdot 13)$ & $1 \cdot 08(1 \cdot 04-1 \cdot 13)$ & $1 \cdot 11(1 \cdot 04-1 \cdot 17)$ \\
\hline Other & $353(5 \cdot 7)$ & $1 \cdot 02(0 \cdot 95-1 \cdot 09)$ & $1 \cdot 04(0 \cdot 97-1 \cdot 11)$ & $1 \cdot 04(0 \cdot 97-1 \cdot 11)$ & $1 \cdot 05(0 \cdot 97-1 \cdot 13)$ \\
\hline
\end{tabular}

* Model 1 adjusted for the following index patient characteristics (age category, HIV status, smoking status, alcohol use) and the following HHC characteristics (age category, elf reported diabetes mellitus, number of BCG scars, alcohol use, nutritional status, socio-economic status by tertile, use of isoniazid preventive therapy and history of previous TB disease. $13 \cdot 5 \%$ of the observations were excluded due to missing data.

**Model 2 adjusted for the factors included in model 1 plus the following characteristics of the index case (presence of cavities of CXR, sputum smear grade and diagnostic delay. $13 \cdot 5 \%$ of the observations were excluded due to missing data.

***Model 3 adjusted for the factors included in model 2 plus the time until initiation of effective therapy. $16.7 \%$ of the observations were excluded due to missing data. 


\begin{tabular}{|c|c|c|c|c|}
\hline Resistance & Univariate analysis & $\begin{array}{c}\text { Multivariate model } \\
1 *\end{array}$ & $\begin{array}{c}\text { Multivariate model } \\
2 * *\end{array}$ & $\begin{array}{c}\text { Multivariate model } \\
3^{* * *} \\
\end{array}$ \\
\hline & $\mathrm{HR}(95 \% \mathrm{CI})^{* * * *}$ & $\mathrm{HR}(95 \% \mathrm{CI})$ & $\operatorname{HR}(95 \% \mathrm{CI})$ & $\mathrm{HR}(95 \% \mathrm{CI})$ \\
\hline Susceptible & 1(Ref) & 1 (Ref) & 1 (Ref) & 1(Ref) \\
\hline Mono-Inh & $0 \cdot 39(0 \cdot 12-1 \cdot 32)$ & $0 \cdot 17(0 \cdot 02-1 \cdot 26)$ & $0 \cdot 16(0 \cdot 02-1 \cdot 12)$ & $0(0$ to $\operatorname{Inf})$ \\
\hline Mono-Strep & $1 \cdot 18(0 \cdot 81-1 \cdot 72)$ & $1 \cdot 18(0 \cdot 78-1 \cdot 77)$ & $1 \cdot 17(0 \cdot 77-1 \cdot 76)$ & $1 \cdot 23(0 \cdot 81-1 \cdot 86)$ \\
\hline Inh+Strep & $0 \cdot 52(0 \cdot 22-1 \cdot 23)$ & $0 \cdot 49(0 \cdot 19-1 \cdot 26)$ & $0 \cdot 48(0 \cdot 19-1 \cdot 24)$ & $0 \cdot 55(0 \cdot 21-1 \cdot 42)$ \\
\hline MDR & $1 \cdot 22(0 \cdot 87-1 \cdot 72)$ & $1 \cdot 28(0 \cdot 9-1 \cdot 83)$ & $1 \cdot 28(0 \cdot 89-1 \cdot 82)$ & $1 \cdot 36(0 \cdot 77-2 \cdot 38)$ \\
\hline Other & $1 \cdot 57(0 \cdot 99-2 \cdot 48)$ & $1 \cdot 79(1 \cdot 09-2 \cdot 93)$ & $1 \cdot 8(1 \cdot 09-2 \cdot 96)$ & $1 \cdot 73(1-3)$ \\
\hline
\end{tabular}

* Model 1 adjusted for the following index patient characteristics (age category, HIV status, smoking status, alcohol use) and the following HHC characteristics (age category, elf reported diabetes mellitus, number of BCG scars, alcohol use, nutritional status, socio-economic status by tertile, use of isoniazid preventive e therapy and history of previous TB disease. $15 \cdot 5 \%$ of the observations were excluded due to missing data.

**Model 2 adjusted for the factors included in model 1 plus the following characteristics of the index case (presence of cavities of CXR, sputum smear grade, and diagnostic delay). $15 \cdot 5 \%$ of the observations were excluded due to missing data.

***Model 3 adjusted for the factors included in model 2 plus the time until initiation of effective therapy. $19 \cdot 3 \%$ of the observations were excluded due to missing data.

$* * * * \mathrm{CI}$ refers to confidence interval 


\begin{tabular}{|l|l|l|}
\hline $\begin{array}{l}\text { Table 4. Risk of genotypically matched incident TB disease } \\
\text { among household contacts of TB patients by Mycobacterium } \\
\text { tuberculosis drug resistance profile. }\end{array}$ \\
\hline Resistance & \multicolumn{1}{|c|}{$\begin{array}{c}\text { HR (95\% CI) } \\
(\mathbf{N}=1009)\end{array}$} & $\begin{array}{c}\text { HR (95\% CI) } \\
\text { (N=10109) }\end{array}$ \\
\hline Susceptible & Ref & Ref \\
\hline Mono-Inh & $0(0-$ Inf $)$ & $0(0-$ Inf) \\
\hline Mono-Strep & $0 \cdot 53(0 \cdot 16-1 \cdot 77)$ & $0 \cdot 45(0 \cdot 14-1 \cdot 51)$ \\
\hline Inh+Strep & $0 \cdot 43(0 \cdot 05-3 \cdot 5)$ & $0 \cdot 38(0 \cdot 05-2 \cdot 99)$ \\
\hline Multidrug & $1 \cdot 13(0 \cdot 51-2 \cdot 5)$ & $0 \cdot 98(0 \cdot 45-2 \cdot 12)$ \\
\hline Other & $1 \cdot 26(0 \cdot 41-3 \cdot 93)$ & $1 \cdot 66(0 \cdot 65-4 \cdot 27)$ \\
\hline
\end{tabular}

*Hazard ratio for having incident TB that is an identical match with the index patient's MIRU patterns

** Hazard ratio for having incident TB that is a relaxed match with the index patient's MIRU patterns 


\begin{tabular}{|c|c|c|c|}
\hline \multirow[t]{2}{*}{ Resistance } & $\begin{array}{c}\text { Univariate } \\
(\mathrm{N}=3608)\end{array}$ & $\begin{array}{c}\text { Multivariate model 1* } \\
(\mathrm{N}=3432)\end{array}$ & $\begin{array}{c}\text { Multivariate model 2*** } \\
(\mathrm{N}=2932)\end{array}$ \\
\hline & RR $95 \%$ CI*** & RR 95\% CI & RR 95\% CI \\
\hline Susceptible & 1 (Ref) & 1 (Ref) & 1 (Ref) \\
\hline Mono-Inh & $1(0 \cdot 73-1 \cdot 37)$ & $1(0 \cdot 73-1 \cdot 37)$ & $0.99(0.72-1.37)$ \\
\hline Mono-Strep & $0.99(0 \cdot 86-1 \cdot 14)$ & $0 \cdot 98(0 \cdot 85-1 \cdot 14)$ & $1(0 \cdot 86-1 \cdot 16)$ \\
\hline Inh + Strep & $1 \cdot 08(0 \cdot 9-1 \cdot 29)$ & $1 \cdot 13(0 \cdot 95-1 \cdot 35)$ & $1 \cdot 11(0 \cdot 92-1 \cdot 34)$ \\
\hline Multidrug & $1 \cdot 27(1 \cdot 15-1 \cdot 42)$ & $1 \cdot 24(1 \cdot 12-1 \cdot 38)$ & $1 \cdot 18(0 \cdot 98-1 \cdot 42)$ \\
\hline Other & $1.02(0 \cdot 81-1 \cdot 29)$ & $1 \cdot 05(0 \cdot 83-1 \cdot 32)$ & $1 \cdot 04(0 \cdot 82-1 \cdot 33)$ \\
\hline
\end{tabular}

*Model 1 adjusted for the following patient characteristics: gender, HIV status, socioeconomic status by tertile, lineage.

**Model 2 adjusted for the factors in Model 1 plus time to effective treatment.

$* * * \mathrm{CI}$ denotes confidence interval 